УДК: 538.911

А. С. Соломатин ${ }^{1}$, Е. В. Царева ${ }^{1}$, В. И. Мащенко ${ }^{2}$ А. В. Савин ${ }^{2}$ В. Г. Чигринов ${ }^{3}$ Д. Н. Чаусов,

НОВЫЕ ПРИНЦИПЫ ОРГАНИЗАЦИИ ИНТЕРАКТИВНОГО МНОГОКАНАЛЬНОГО ВИЗУАЛЬНОГО ИНФОРМАЦИОННОГО ПОТОКА ДИСПЛЕЙНЫМИ И ПРОЕКЦИОННЫМИ СРЕДСТВАМИ НА ОСНОВЕ УПОРЯДОЧЕННЫХ КРИСТАЛЛИЧЕСКИХ МИКРОСТРУКТУР 4-ЦИАНО-4-ОКТИЛОКСИБИФЕНИЛА В БОРОСИЛОКСАНОВЫХ ГЕЛЯХ

\author{
${ }^{1}$ Российский химико-технологический университет им. Д. И. Менделеева, \\ Миусская площадь, д. 9, 125047 Москва, Россия \\ ${ }^{2}$ Московский государственный областной университет (МГОУ), \\ ул. Веры Волошиной, д. 24, 141014 Мытищи, Россия \\ ${ }^{3}$ School of Physics and Optoelectronic Engineering Foshan University, \\ Foshan, Guangdong 528000, China. E-mail: eechigr@ust.hk \\ ${ }^{4}$ Национальный исследовательский технологический университет «МИСиС», \\ Ленинский проспект, д. 4, 119049 Москва, Россия \\ E-mail: Sotrudnica_UNC@mail.ru
}

\begin{abstract}
Актуально развитие больших экранов коллективного назначения в направлении обеспечения каждого зрителя (группь совместно расположенных зрителей) видимым только ими (в их угловом диапазоне относительно экрана) изображением - визуальным информационным потоком - на всей поверхности экрана как альтернатива разбиению экрана на отдельные области с различными отображаемыми картинками. Важно обеспечить возможность регулировать упомянутые угловые диапазоны - области просмотра индивидуального изображения. Важна также и возможность переключать дисплей в обычный режим коллективного просмотра с очень высокой разрешающей способностью. $B$ данной работе изложены конструктивные решения $u$ предложены жидкокристаллические (ЖК) материалы для решения данной задачи, а также, весьма сходным образом, предложены новые решения для формирования проекционного изображения коллективного просмотра. Предложена конструкиия основного элемента дисплея - пикселя, формирующего требуемое для решения поставленной задачи пространственное распределение световых потоков, а также аналогичная конструкция пикселя просветного матричного проектора. Микроструктуры ЖК 4-циино-4октилоксибифенил (8ОСВ) различной (заданной заранее) геометрии в боросилоксановых (БС) матрицах весьма перспективны - резкое охлаждение ниже температуры плавления $\left(55^{\circ} \mathrm{C}\right)$ приводит $\kappa$ кристаллизации ЖК и долговременному фиксированию полученных микроструктур (от долей до десятков микрон). Необходимо ЖК-композит, нагретый выме температуры плавления (структуры из ориентированных кристаллитов переходят в ЖК-фазу), сначала сориентировать приложением электрического поля, пространственно распределенного по некоторому заранее заданному правилу, а затем зафиксировать ориентированное состояние охлаждением при помощи кристаллизации ЖК.
\end{abstract}

Ключевые слова: жидкие кристаллы, 4-ииано-4-октилоксибифенил (8ОСВ), боросилоксановые гели, жидкокристаллические композиты, дисплей, проектор.

DOI: $10.18083 /$ LCAppl.2020.3.57

(С Соломатин А. С., Царева Е. В., Мащенко В. И., Савин А. В., Чигринов В. Г., Чаусов Д. Н., 2020 
A. S. Solomatin ${ }^{1}$, Ye. V. Tsareva ${ }^{1}$, V. I. Mashchenko ${ }^{2}$, A. V. Savin ${ }^{2}$, V. G. Chigrinov ${ }^{3}$, D. N. Chausov ${ }^{2,4}$

\title{
NEW PRINCIPLES OF ORGANIZING AN INTERACTIVE MULTI-CHANNEL VISUAL INFORMATION FLOW BY DISPLAY AND PROJECTIVE MEANS ON THE BASIS OF ORDERED CRYSTALLINE 4-CYANO-4-OCTYLOXYBIPHENYL MICROSTRUCTURES IN BOROSILOXANE GELS
}

\author{
${ }^{1}$ D. Mendeleev University of Chemical Technology of Russia \\ 9 Miusskaya square, Moscow, 125047, Russia. E-mail: Sotrudnica_UNC@mail.ru \\ ${ }^{2}$ Moscow Region State University, 24 Very Voloshinoy St., Mytishi, 141014, Russia \\ ${ }^{3}$ School of Physics and Optoelectronic Engineering Foshan University, \\ Foshan, Guangdong 528000, China. E-mail: eechigr@ust.hk \\ ${ }^{4}$ National University of Science and Technology «MISIS», \\ 4 Leninsky Prospekt, Moscow, 119049, Russia
}

The development of large collective screens in ordert to provide each viewer (group of jointly located viewers) with a visual information flow that is an image that is visible only to them (in their angular range relative to the screen) on the entire surface of the screen is a topical direction of investigations. This task can be considered as an alternative to dividing the screen into separate areas with different displayed images. It is important to provide the ability to adjust the mentioned angular ranges - the viewing area of the individual image. The ability to switch the display to normal collective viewing mode with a very high resolution is also essential option. To solve the assigned above task, constructive solutions are stated and liquid crystal (LC) materials are suggested. In addition, new solutions for the formation of a projection image of collective viewing are proposed. A design of the main display element (a pixel), which forms the spatial distribution of the light flux required to solve the problem is proposed. A similar pixel design of the translucent matrix projector is also developed. Microstructures of LC 4-cyano-4-octyloxybiphenyl (8OCB) of various (predefined) geometries in borosiloxane $(B S)$ matrices are very promising. The abrupt cooling below the melting temperature $\left(55{ }^{\circ} \mathrm{C}\right)$ leads to crystallization of $L C$ and the obtained microstructures (from fractions to tens of microns) undergo long-term fixation. At first, the LC composite needs to be heated above melting temperature (at this stage the structures of oriented crystallites transfer into the LC phase) and oriented by applying an electric field spatially distributed according to some predetermined rule. Then, the oriented state can be fixed by cooling due to crystallization of the $L C$.

Key words: liquid crystals, 4-cyano-4-octyloxybiphenyl (8OCB), borosiloxane gels, liquid crystal composites, display, projector.

\section{Введение}

В настоящее время наблюдается тенденция к широкому распространению в самых различных видах деятельности (управление: технологическими процессами - диспетчерские; транспортом - кабина вертолета, самолета, речного и морского транспорта, железнодорожного транспорта, метро; развлекательные мероприятия, клубы, рестораны; домашний досуг фильмы и игры; офис - обработка информации финансовых рынков; многое иное) больших экранов для коллективного просмотра, использование жидких кристаллов (ЖК) обеспечило их углы обзора [1], быстроту смены кадров, контрастность, энергоэкономность, как и было предсказано в [2-4]. Однако именно вследствие широкого распространения стали очевидны их принципиальные недостатки, главным из которых является проблема различных информационных (визуальных) потоков, в которых заинтересованы различные группы и отдельные зрители.

Разбиение коллективного экрана на части (рис. 1) с информацией для различных потребителей в значительной степени обесценивает сам принцип его наличия и сопровождается более часто переключаемым интерактивным персональным экраном настольным на рабочем месте (рис. 1). 


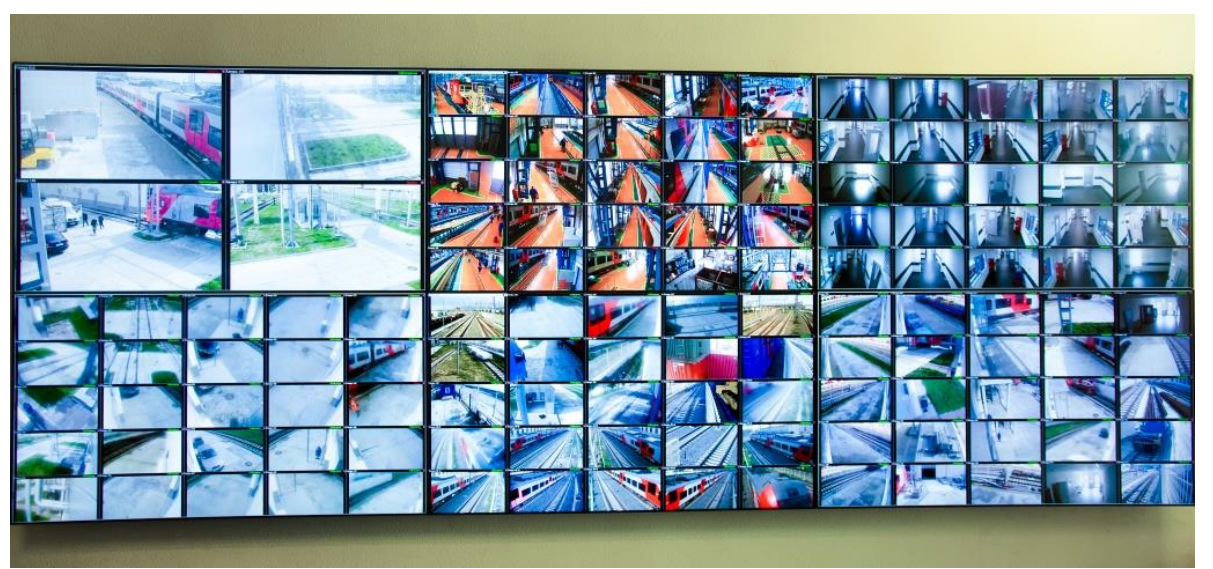

Рuc. 1. Разбиение коллективного экрана на части с информацией для различных потребителей сопровождается интерактивным персональным экраном на рабочем месте

Fig. 1. The splitting of the collective screen into parts with information for individual consumers is accompanied by an interactive personal screen at the workplace

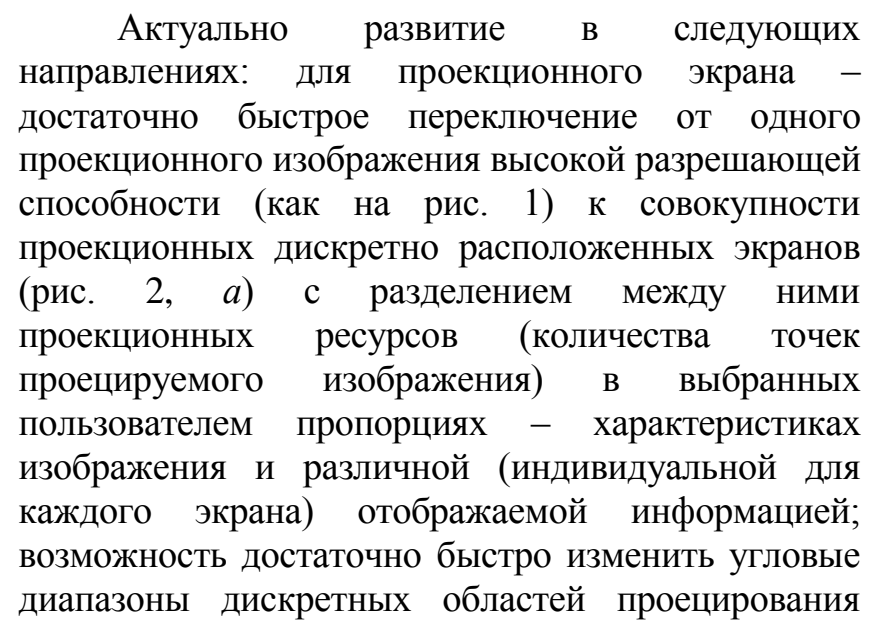

(расположение проекционных изображений на различных поверхностях в различных, относительно проектора, направлениях); для дисплея - достаточно быстрое переключение от одного изображения высокой разрешающей способности (как на рис. 1) с углом обзора максимально широким (вплоть до $180^{\circ}$ ) к совокупности дискретно расположенных (смежных друг другу) угловых диапазонов - областей демонстрации индивидуально различного видеоряда (рис. 2, б); возможность достаточно быстро перерегулировать границы областей просмотра индивидуально различного видеоряда.

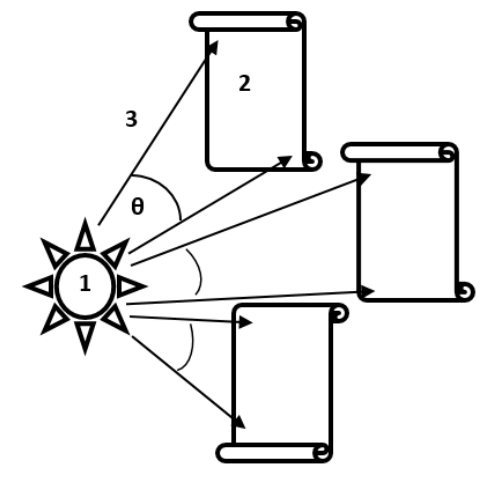

$a$

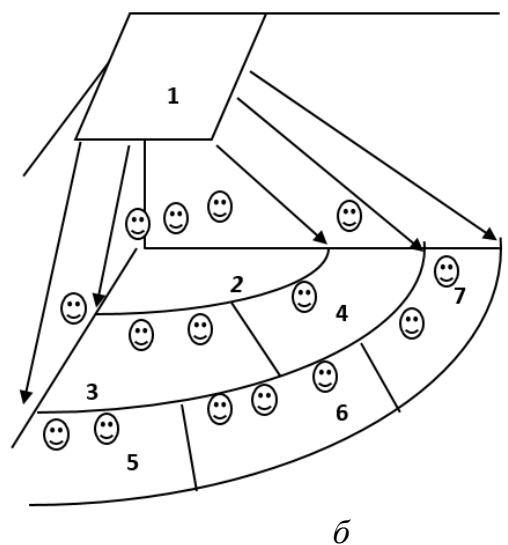

$\sigma$

Puc. 2, a: 1 - проектор, 2 - экран и соответствующие угловые диапазоны проецирования, 3 - край одного из угловых диапазонов, $\theta$ - угловой диапазон; б: 1 - дисплей, 2, 3, 4, 5, 6, 7 - угловые диапазоны отображения индивидуально различного видеоряда и группы зрителей

Fig. 2, a: - 1 - projector, 2 - screen and corresponding projection angular ranges, 3 - edge of one of the angular ranges, $\theta$ - angular range; $b: 1$ - display, 2, 3, 4, 5, 6, 7 - angular display ranges of individually different footage and a group of viewers 


\section{Пиксель}

Данная работа является дальнейшим развитием [5-9], объединяя изложенные в них разрозненно конструктивные решения, режимы использования, ЖК-композитные материалы. Дополняя [5-9], предлагаем новый вариант пикселя дисплея, и такая же конструкция может быть у пикселя матрицы просветного проектора. Именно его конструкция (рис. $3, a$ ), использующая свойства предложенных в [7-9] ЖК-композитных материалов, позволяет переключаться между режимами.

Световой поток от источника света идет через несколько (принимающие световые потоки от источника в разных направлениях - угловых диапазонах) регуляторов интенсивности пропускания. Отрегулированный по интенсивности световой поток (несколько потоков в различных направлениях - угловых диапазонах, с индивидуально отрегулированными интенсивностями) идет на оптический элемент, из которого он выйдет с достаточно равномерной плотностью в заданном угловом диапазоне - туда, где его увидят зрители как пиксель дисплея - точку изображения, часть видеокартинки для находящихся в данном угловом диапазоне (рис. 2, б). Остальные световые потоки (рис. 3, a) им будут не видны, так как каждой пространственно локализованной группе зрителей (рис. 2, б) посылает световой поток только один субэлемент пикселя (рис. $3, a$ ).

Упомянутый оптический элемент, из которого световой поток выйдет с достаточно равномерной плотностью в заданном угловом диапазоне, - это линза ЖК [10], управляемая электрическим полем (рис. 3, б). Рисунок 3, б весьма схематичен, реальная картина значительно сложнее. Линзы ЖК, управляемые электрическим полем, достаточно широко применяются в различных устройствах (фото, видео, и т. д.) .

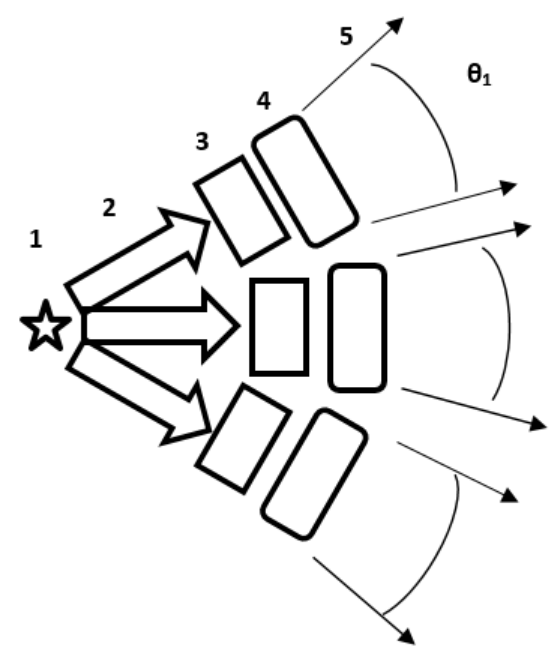

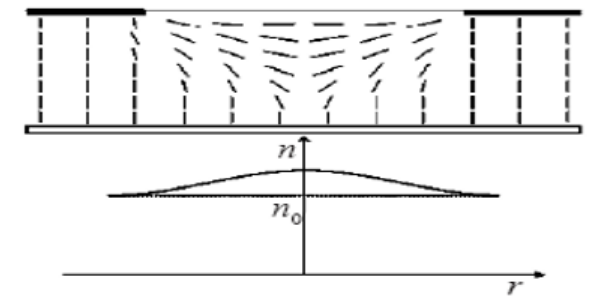

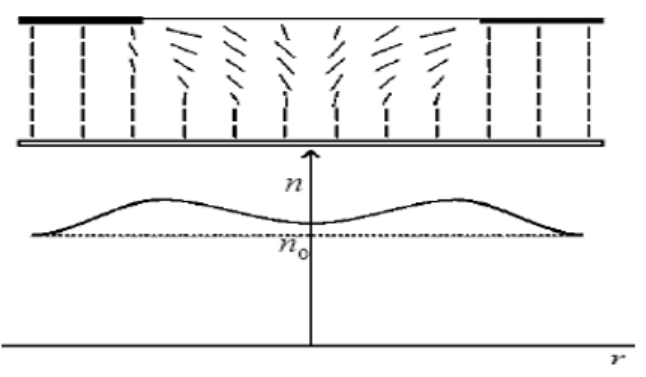

$\sigma$

Puc. 3, a- пиксель: 1 - задняя подсветка (например, светодиод), 2 - световые потоки от источника на регуляторы яркости (интенсивности), 3 - регулятор яркости (например, твист-ячейка), 4 - линза ЖК, регулирующая направление выходящего светового потока, 5 - край выходящего светового потока, $\theta_{1}$ - угловая ширина выходящего светового потока; б - ЖК-линза, управляемая электрическим полем, сверху собирающая (фокусирующая), снизу рассеивающая (расфокусирующая)

Fig. 3. $a$-pixel: 1 - backlight (for example, an LED), 2 - light fluxes from the source to the brightness (intensity) controls,

3 - brightness control (for example, a twist-cell), 4 - LCD lens, regulating the direction of the outgoing light flux, 5 - edge of the outgoing luminous flux, $\theta_{1}$ - angular width of the outgoing light flux; $b$ - LCD lens, controlled by an electric field, collecting (focusing) from above, scattering (defocusing) from below 


\section{ЖК-композиты}

Направленное управляемое микроструктурирование ЖК-соединений под конкретную задачу является одним из современных трендов в построении сложных оптических систем [7-9, 11, 12].

В [7-9, 11] предложены ЖК-композитные материалы, актуальные для использования в оптических устройствах. В частности, в [7] предложен ЖК-композит 4-ицано-4-октилоксидифенил (8ОСВ) в боросилоксане (БС). Он замечателен тем, что резкое охлаждение ниже температуры плавления $8 O C B\left(55^{\circ} \mathrm{C}\right)$ приводит к кристаллизации ЖК и долговременному фиксированию полученных в боросилоксановых матрицах микроструктур различной (заданной заранее) геометрии (размером от долей до десятков микрон). Практически это означает, что линза ЖК на основе микроструктуры ЖК $8 O C B$ в боросилоксане - микрокапле заданной заранее (при изготовлении линзы) геометрии - при помощи приложенного электрического поля регулируется под заданные требования (формирует необходимые параметры выходного светового потока), после чего остывает (отключается подогрев - терморегуляция) и, как описано выше, фиксируется на длительное время, т. е. энергонезависимо (без приложения поля) функционирует с постоянными параметрами (постоянный угловой диапазон, в котором зрители увидят их индивидуальный видеоряд). По мере возникновения необходимости перерегулировать пространственное распределение световых потоков дисплея (и, соответственно, пикселя) изменить границы угловых диапазонов, в которых демонстрируются различные видеопотоки, цикл повторяется, т. е. линза ЖК подогревается, перерегулируется приложенным полем, остывает, ее параметры фиксируются на длительное время.

Уникальные свойства ЖК-композитам придает используемый материал - БС, который является неньютоновской жидкостью и может находиться в зависимости от времени приложения внешней фиксированной нагрузки в различных агрегатных состояниях. При низких временах в состоянии жидкости, с уменьшением времени прикладываемого импульса он переходит в высокоэластическое состояние и далее в твердое упругое состояние. БС представляет собой материал, содержащий участки, образованные олигомерными молекулами с цепочками из полидиметилсилоксановых звеньев и группами подобными боросиликатным стеклам, связанными посредством водородных связей. Боросиликатные группы подвержены механическому застекловыванию в соответствии с принципом температурно-временной суперпозиции, в то время как силоксановые части БС остаются подвижными до некоторых времен воздействия внешнего импульса. Таким образом, БС при механическом воздействии, делающим его упругим, можно представить как трехмерную сетку, узлами которой являются механически застеклованные участки. На ощупь БС напоминают жевательную резинку. Из него легко можно скатать шарик или придать любую другую форму. При помещении на твердую поверхность образец не держит форму и растекается. При резком растяжении БС рвется, при медленном растягивается. От пола БС отскакивает, как резиновый мячик.

Свойствами БС можно управлять при помощи изменения соотношения исходных компонентов, а также температуры синтеза. На рис. 4 представлена микрофотография БС матрицы, сделанной в поляризационном оптическом микроскопе (ПОМ).

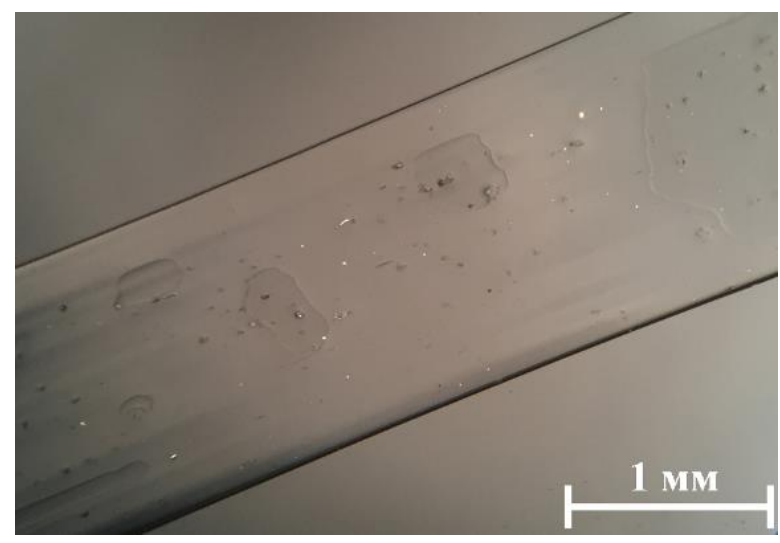

Рис. 4. Микрофотография ПОМ пленки БС, полученного при температурах свыше $200{ }^{\circ} \mathrm{C}$. Поляризаторы скрещенные

Fig. 4. POM microphotograph of BS film obtained at $200{ }^{\circ} \mathrm{C}$. The polarizers are crossed

Хорошо видно, что БС является прозрачным однородным и не двулучепреломляет. 
Для получения ЖК-композита на основе БС и ЖК были взяты полидиметилсилоксан с концевыми гидроксильными группами (ПДМС), марки СКТН А (молекулярная масса 20000 г/моль) и борная кислота (БК). Соотношение компонентов было подобрано экспериментально, чтобы обеспечить хорошие эластические характеристики композита и в то же время сохранить его оптическую прозрачность. Наилучшее подобранное соотношение компонентов ПДМС к БК составило 20:1. Для синтеза БС реакционная смесь механически перемешивалась в стеклянном химическом стакане и переносилась в температурную ячейку. С помощью ротационной вискозиметрии на вискозиметре Viscotester $E$ («Thermoscientific», Германия). была исследована скорость реакции между ПДМС и БК в диапазоне температур до $100{ }^{\circ} \mathrm{C}$. Показано, что с ростом температуры растет скорость реакции. Если при комнатной температуре для достижения вязкости более $6 \cdot 10^{6} \mathrm{M}$ Па $\mathrm{c}$ необходимо около 24 часов, то при $100^{\circ} \mathrm{C}$ вязкость нарастает менее чем за 30 мин.

Введение ЖК в БС осуществляли путем механического перемешивания при комнатной температуре. ЖК вводили в БС в состоянии мезофазы в различных концентрациях. С помощью ПОМ охарактеризована микроструктура образцов (рис. 4).

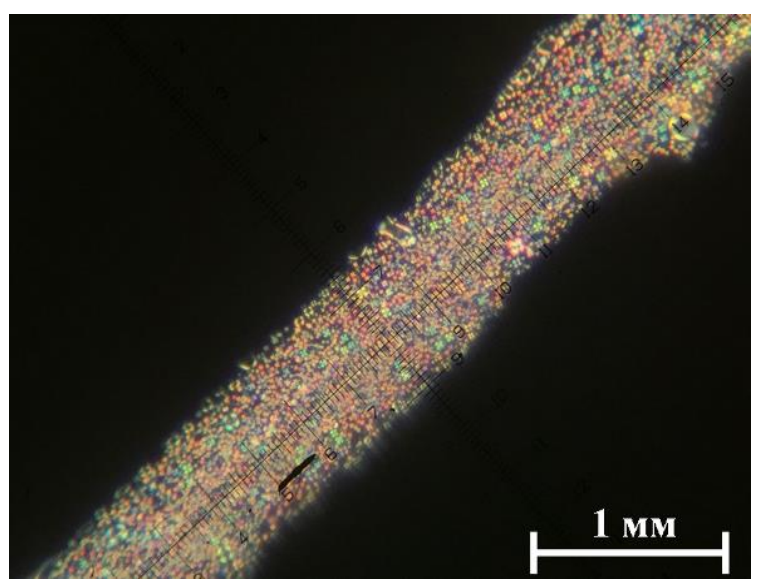

Puc. 5. Микрофотография ПОМ ЖК-композита на основе БС и 8ОСВ с концентрацией ЖК 5 мас. \%. Поляризаторы скрещенные

Fig. 5. POM microphotograph of the LC composite based on $\mathrm{BS}$ and $8 \mathrm{OCB}$ with the $\mathrm{LC}$ concentration of 5 wt. $\%$. The polarizers are crossed
На рисунке 5 хорошо видно, что образец имеет микрогетерогенную структуру, состоящую из двулучепреломляющих капель ЖК, внедренных в БС-матрицу.

Смешение ЖК с полученным БС практически не влияет на характер фазовых переходов ЖК, что было показано методом дифференциальной сканирующей калориметрии. Из полученных ЖКкомпозитов формировали образцы в виде пленок шириной от 1 мм до $1 \mathrm{~cm}$, которые могут быть использованы в качестве материалов для элементов конструкции представленного оптического устройства. Управление формой капель может быть осуществлено путем ориентации БС в высокоэластическом состоянии, а фиксирование структуры - при помощи охлаждения системы [7]. Обратный процесс задается нагреванием с самопроизвольным превращением микролинз в микрокапли. Для осуществления таких процессов интерес представляет создание микроманипуляторных систем (например, на основе материалов с памятью формы), снабженных системами подогрева и охлаждения со скоростями быстродействия, необходимыми для реализации работы оптического устройства. Интерес представляет получение подобных систем на основе производных порфина, фталоцианина и холестерола [13-16].

\section{ЖК-дисплей}

В зависимости от потребностей зрителей ЖК-дисплей перерегулируется (переключается) в режим:

- обычного коллективного просмотра - каждый пиксель посылает световые потоки каждым своим субэлементом в максимально широком (вплоть до $180^{\circ}$ ) угловом диапазоне, что означает участие в формировании изображения всех субэлементов пикселя в качестве точек изображения, т. е. максимальная разрешающая способность дисплея;

- просмотра индивидуальных видеопотоков зрителями в заданных угловых диапазонах (не пересекающихся), каждый зритель (группа зрителей) видит только один - направленный именно к ним - поток видеоинформации.

Переключение между режимами и/или изменение угловых диапазонов индивидуального просмотра непродолжительно (менее минуты). 


\section{Проектор}

В зависимости от потребностей зрителей проектор перерегулируется (переключается) в режим:

- обычного просмотра проекционного изображения - каждый пиксель посылает световые потоки каждым своим субэлементом в угловом диапазоне, соответствующем угловой величине точки проекционного изображения, что означает максимальное число точек изображения - его наиболее высокую разрешающую способность;

- просмотра дискретных проекционных изображений на различных - не соприкасающихся границами (хотя это и не обязательно) поверхностях проецирования, количество которых соответствует количеству субэлементов пикселя, каждое проекционное изображение состоит из одинакового количества точек - равного числу пикселей;

- просмотра дискретных проекционных изображений на различных - не соприкасающихся границами (хотя это и не обязательно) поверхностях проецирования, количество которых не больше чем количество субэлементов пикселя, отдельное проекционное изображение состоит из количества точек, равного или кратного числу пикселей (в каждом пикселе в формировании какого-то одного определенного изображения участвует одинаковое число субэлементов).

\section{Выводы}

В работе предложены новые конструктивные решения, жидкокристаллические материалы и принципы управления световым потоком при формировании изображений.

Дополнен предложенный ранее $[5,6]$ новый путь развития больших экранов коллективного назначения в направлении обеспечения каждого зрителя (группы совместно расположенных зрителей) видимым только ими (в их угловом диапазоне относительно экрана) изображением визуальным информационным потоком - на всей поверхности экрана как альтернатива разбиению экрана на отдельные области с различными отображаемыми картинками.

Впервые предложена возможность регулировать угловые диапазоны - области просмотра индивидуального изображения.
Впервые предложена возможность переключать дисплей в обычный режим коллективного просмотра с очень высокой разрешающей способностью.

Впервые предложена конструкция основного элемента дисплея - пикселя, формирующего требуемое для решения поставленной задачи пространственное распределение световых потоков, а также аналогичная конструкция пикселя просветного матричного проектора.

Предложены полученные ранее [7] жидкокристаллические (ЖК) материалы для решения задач, рассмотренных в данной работе.

Исследование выполнено за счет гранта Российского научного фонда (проект № 20-19-00201).

\section{Список литературы / References}

1. Dadivanyan A.K., Belyaev V.V., Chausov D.N., Stepanov A.A., Smirnov A.G., Tsybin A.G., Osipov M.A. Nanomesh Aluminum Films for LC Alignment. Theoretical and Experimental Modeling. Mol. Cryst. Liq. Cryst., 2015, 611, 117.

2. Пикин С. А., Блинов Л. М. Жидкие кристаллы. М. : Наука, 1982. 208 c. [Pikin S.A., Blinov L.M. Liquid crystals. M. : Nauka, 1982, 208 p. (in Russ.)].

3. Chausov D.N., Kurilov A.D., Belyaev V.V., Kumar S. Parameters of LC molecules' movement measured by dielectric spectroscopy in wide temperature range. Opto-Electronics Review, 2018, 26 (1), 44.

DOI: $10.1016 /$ j.opelre.2017.12.001

4. Dadivanyan A.K., Chausov D.N., Belyaev V.V., Bugaev A.S. The entropy effect on liquid crystal orientation relative to a nanopore surface. Doklady Physics, 2014, 59, 457.

DOI: $10.1134 /$ S1028335814100115.

5. Соломатин А. С., Беляев В. В. Дисплей с многопользовательским направленным распределением визуальной информации и проектор с управляемым распределением луча света на основе жидких кристаллов с неоднородным распределением директора // Вестник Московского гос. обл. ун-та. Серия: Физика-математика. 2018. T. 1. C. 47-62. [Solomatin A.S., Belyaev V.V. Display with multi-user directional distribution of visual information and a projector with a controlled distribution of the light beam based on liquid crystals with an inhomogeneous director distribution. Bull. of the Moscow Region State University, 2018, 1, 47-62 (in Russ.)]. 
6. Соломатин А. С. Дисплей с многопользовательским индивидуально-различным отображением. Управляемое распределение проецируемого светового потока проектором на основе жидких кристаллов // Вестник Московского гос. обл. ун-та. Серия: Физика-математика. 2018. T. 2. C. 34-44. [Solomatin A.S. Display with multiuser individually different display. Controlled distribution of projected luminous flux by a liquid crystal projector. Bull. of the Moscow Region State University, 2018, 2, 34-44 (in Russ.)].

7. Ермакова М. В., Мащенко В. И., Чаусова О. В., Соломатин А. С., Волосникова Н. И., Чаусов Д. Н. Формирование упорядоченных кристаллических микроструктур 4-циано-4-октилоксидифенила в боросилоксановых гелях // Жидк. крист. $и$ ux практич. использ. 2019. Т. 19, № 4. С. 61-66. [Ermakova M.V., Mashchenko V.I., Chausova O.V., Solomatin A.S., Volosnikova N.I., Chausov D.N. Formation of ordered crystalline microstructures of 4cyano-4-octyloxydiphenyl in borosiloxane gels. Liq. Cryst. and their Appl., 2019, 19 (4), 61-66. (in Russ.). DOI: $10.18083 /$ LCAppl.2019.4.61].

8. Соломатин А. С., Мащенко В. И., Шашкова Ю. О., Беляев В. В. Особенности формирования микроструктуры и оптические свойства жидкокристаллических композитных твист-ячеек // Вестник Московского гос. обл. ун-та. Серия: Физика-математика. 2017. Т. 2. С. 53-63. [Solomatin A.S., Mashchenko V.I., Shashkova Yu.O., Belyaev V.V. Features of formation of the microstructure and optical properties of liquid crystal composite twist cells. Bull. of the Moscow Region State University, 2017, 2, 53-63 (in Russ.).

DOI: $10.18384 / 2310-7251-2017-2-53-63$ ],

9. Мащенко В. И., Шашкова Ю. О., Соломатин А. С., Беляев В. В. Особенности формирования микроструктуры жидкокристаллических композитов на основе боросилоксана // Вестник Московского гос. обл. ун.-та. Серия: Физика-математика. 2017. T. 2. C. 34-45 [Mashchenko V.I., Solomatin A.S., Shashkova Yu.O., Belyaev V.V. Peculiarities of the formation of a microstructure of borosiloxane liquid crystal composites. Bull. Of the Moscow Region State University. 2017, 2, 34-45 (in Russ.).

DOI: $10.18384 / 2310-7251-2017-2-34-45]$.

10. Невская Г. Е., Томилин М. Г. Адаптивные линзы на основе жидких кристаллов // Оптический журнал. 2008. T. 75, №. 9. C. 35-48. [Nevskaya G.E., Tomilin M.G. Adaptive lenses based on liquid crystals. Journal of Optical Technology, 2008, 75 (9), 35-48. (in Russ.)].
11. Патент № 0002607454. Способ получения смеси жидкого кристалла с полимером для дисплейной техники и оптоэлектроники / В. В. Беляев, В. И. Мащенко, А. С. Соломатин, Д. Н. Чаусов; Приоритет от 27.04.2015 [RF patent 2607454. A method of obtaining of amixture of liquid crystal with a polymer for display technology and optoelectronics / V.V. Belyaev, V.I. Mashchenko, D.N. Chausov, A.S. Solomatin; Priority 27.04.2015 (in Russ.)].

12. Васильчикова Е. Н., Константинов М. С., Мащенко В. И., Кучеров Р. Н., Чаусов Д. Н., Дадиванян А. К. Особенности процесса кристаллизации 4,4'азоксианизола в виде множественных «кофейных колец» // Жидк. крист. и их практич. использ. 2020. T. 20, № 1. C. 47-52. [Vasilchikova E.N., Konstantinov M.S., Mashchenko V.I., Kucherov R.N., Chausov D.N., Dadivanyan A.K. Specific features of crystallization process of 4,4-azoxyanisole in the form of multiple «coffee rings». Liq. Cryst. and their Appl., 2020, 20 (1), 47-52. (in Russ.).

DOI: $10.18083 /$ LCAppl.2020.1.47].

13. Chausov D.N., Kurilov A.D., Kazak A.V., Smirnova A.I., Velichko V.K., Gevorkyan E.V., Rozhkova N.N., Usol'tseva N.V. Dielectric properties of liquid crystalline composites doped with nano-dimensional fragments of shungite carbon. Liq. Cryst., 2019, 46, 1345-1352. DOI: 10.1080/02678292.2019.1566503.

14. Usol'tseva N., Bykova V., Zharnikova N., Alexandrov A., Semeikin A., Kazak A. Influence of mesosubstituted porphyrins molecular structure on their mesogenity. Mol. Cryst. Liq. Cryst., 2010, 525 (1), 184-193. DOI: 10.1080/15421401003799557.

15. Chausov D.N., Kurilov A.D., Kazak A.V., Smirnova A.I., Belyaev V.V., Gevorkyan E.V., Usol'tseva N.V. Conductivity and dielectric properties of cholesteryl tridecylate with nanosized fragments of fluorinated grapheme. J. Mol. Liq., 2019, 291, 111259-7.

16. Usol'tseva N.V., Smirnova A.I., Kazak A.V., Giricheva N.I., Galanin N.E., Shaposhnikov G.P., Bodnarchuk V.V., Yablonskii S.V. Mix-substituted phthalocyanines of a "push-pull"-type and their metal complexes as prospective nanostructured materials for optoelectronics. Opto-Electronics Review, 2017, 25 (2), 127-136. DOI: 10.1016/j.opelre.2017.03.003.

Поступила 12.05.2020 2. Received 12.05.2020

Принята 27.08.2020 г. Accepted 27.08.2020 